\title{
AFECTACIONES OCASIONADAS POR DERRAME DE LODO ACTIVADO OCURRIDO EN EL SECTOR LA INDEPENDENCIA DEL CANTÓN QUININDÉ
}

\author{
AUTORES: William Johao Paredes Álava ${ }^{1}$ \\ Lisseth StefaniaVásquez Alarcón ${ }^{2}$ \\ Genny Dayanara Cortez Quiñonez ${ }^{3}$ \\ Luis Fernando Lucio Villacreses ${ }^{4}$
}

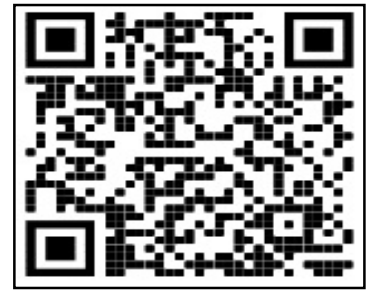

\section{DIRECCIÓN PARA CORRESPONDENCIA: cortez-genny8663@unesum.edu.ec}

Fecha de recepción: 12/08/2020

Fecha de aceptación: 26/11/2020

\section{RESUMEN}

El presente trabajo se realizó en el cantón Quinindé con la finalidad de conocer las afectaciones ocasionadas por el derrame de lodo activado ocurrido en el sector la Independencia que se suscitó tras la ruptura del geo tubo contenedor de lodos sedimentado por parte de la extractora SOPALIN S.A, esta empresa se dedica a extraer aceite crudo vegetal sin refinar de palma africana. La investigación tiene como propósito conocer el nivel de contaminación del río y quienes se vieron afectados. Los métodos utilizados fueron: analítico y cuantitativo. Es importante detallar que los principales resultados encontrados son la contaminación media alta de la zona con una cantidad de $30000 \mathrm{yd}^{3}$. Tal incidente contaminó el río blanco en un $42.9 \%$ situado cerca de la fábrica, dañando y desplazando flora y fauna de la zona en un $85.7 \%$, perjudicando también a agricultores del lugar por las pérdidas económicas acontecidas, mediante la proyección porcentual que se realizó se refleja que la población ha tenido un crecimiento notable al pasar de los años ya que la zona ha tenido un buen desarrollo y aumento su población desde el primer censo que fue en el 1999 donde se contabilizó 62.680 habitantes en todo el cantón y ya para el 2010 su crecimiento fue del $41 \%$ más de la población, llegando a concluir que este desastre ambiental generó falencias en actividades de moradores, y al entorno natural.

PALABRAS CLAVE: investigación, palma africana; contaminación; impacto ambiental.

\section{CONDITIONS CAUSED BY SPILLAGE OF ACTIVATED SLUDGE OCCURRED IN THE INDEPENDENCE SECTOR OF THE QUININDÉ CANTON}

\footnotetext{
${ }^{1}$ Estudiante de la Carrera de Ingeniería Ambiental de la Universidad Estatal del Sur de Manabí. Jipijapa, Manabí, Ecuadorcortez-genny8663@unesum.edu.ec ORCID ID: https://orcid.org/0000-0001-8839-6334

${ }^{2}$ Estudiante de la carrera de Ingeniería Ambiental de la Universidad Estatal del Sur de Manabí Jipijapa, Manabí, Ecuador. paredes-william3172@unesum.edu.ec ORCID ID: https://orcid.org/0000-0002-2472-5853

${ }^{3}$ Estudiante de la carrera de Ingeniería. Ambiental. Jipijapa, Manabí, Ecuador. vasquez-lisseth4308@unesum.edu.ec ORCID ID: https://orcid.org/0000-0002-9147-1590

${ }^{4}$ Ing. Forestal, Magister en Educación y Desarrollo Social, Docente de la Carrera de Ingeniería Ambiental de la Universidad Estatal del Sur de Manabí. Jipijapa, Manabí, Ecuador. luis.lucio@unesum.edu.ec ORCID ID: https://orcid.org/0000-0002-3757-7183
} 
William J. Paredes Álava, Lisseth S.Vásquez Alarcón, Genny D. Cortez Quiñonez, Luis F. Lucio Villacreses

\begin{abstract}
This work was carried out in the Quinindé cantón in order to know the effects caused by the activated sludge spill that occurred in the Independencia sector that arose after the rupture of the sedimented sludge container geo-tube by the extractor SOPALIN SA, This company is dedicated to extracting crude unrefined vegetable oil from African palm. The purpose of the investigation is to know the level of contamination of the river and who was affected. The methods used were: analytical and quantitative. It is important to detail that the main results found are the mediumhigh contamination of the area with an amount of 30,000 $\mathrm{yd}^{3}$. This incident contaminated the white river by $42.9 \%$ located near the factory, damaging and displacing flora and fauna of the area by $85.7 \%$, also harming local farmers due to the economic losses that occurred, through the percentage projection that was carried out. It reflects that the population has had a notable growth over the years since the area has had a good development and its population increased since the first census, which was in 1999 where 62,680 in habitants were counted throughout the canton and already by 2010 its growth was $41 \%$ more than the population, reaching the conclusion that this environmental disaster generated shortcomings in the activities of residents, and the natural environment.
\end{abstract}

KEYWORDS: research, African palm, contamination, environmental impact.

\title{
INTRODUCCIÓN
}

Los cultivos de palma africana predominan en la presente investigación ya que las extractoras en el país causan gran contaminación al elaborar los aceites, estas son vulnerables a accidentes como los derrames de lodos activados cuando no se le da mantenimiento adecuado a sus tuberías, como fue lo sucedido por parte de la extractora SOPALIN S.A. del sector la Independencia. Los países que cultivan dichas plantaciones son África de ahí proviene el nombre de palma africana, Indonesia, Borneo, Costa Rica, y también se encuentra América Latina. Estos cultivos han estado sustituyendo con el $40 \%$ a boques tropicales, mientras que con un $32 \%$ a pastos naturales. La última encuesta dada por la Superficie y producción agropecuaria en 2016 arroja que Ecuador cuenta con 319000 hectáreas sembradas, por otra parte la expansión que ha tenido en Ecuador la plantación de palma africana es una de los factores causantes de la deforestación (Barcelona, U.U. A. 2017.).

Los primeros cultivos de palma aceitera en Ecuador iniciaron en el año 1950 específicamente en Santo Domingo de los Tsáchilas, Quinindé y la zona sur de Esmeraldas, pero fue en los 70 y 80 que empezó a expandirse con fuerza. Actualmente Ecuador ocupa el segundo lugar en Latinoamérica, después de Colombia en producir aceite de palma, mundialmente es el séptimo productor. En la actualidad existen 319000 hectáreas de palma aceitera en Ecuador produciendo 577000 toneladas, de la el 39\% es para consumo nacional, mientras que el $61 \%$ se exporta. La mayor parte de la producción de palma se encuentra en Quinindé con 1900 productores, cabe mencionar que catorce de cuarenta molinos se encuentran en Quinindé, las más nombradas son: grupo La Fabril (Extractora Río Manso), Palcien, Palma de los Andes, Epacem SA, y SOPALIN S.A.(Flacso, 2011).

Las plantas extractoras de aceites son de gran relevancia, puesto que con la ayuda de estas se procesan diferentes tipos como el denominado aceite de palma, si bien es cierto toda empresa

194 UNESUM-Ciencias. Publicación cuatrimestral. Vol. 4, No. 4 (Septiembre-Diciembre), Año 2020. 
debe tener un compromiso tanto social como ambiental hacia la población, es decir precautelar por el bienestar tanto de la industria como a los moradores en caso de dar un acontecimiento que perjudique al entorno natural de donde esta se encuentra ubicada. Las afectaciones dadas por el derrame de lodo activado de aceite de palma fue un incidente que causo daños materiales, económicos, sociales y medioambientales (Municipalidad de Quinindé, 2019). De acuerdo a datos emitidos por el Ministerio del Ambiente en el año 2014 y 2016 Ecuador mostró deforestación con un promedio de 94353 hectáreas por año, la provincia más afectada fue Esmeraldas con 13655 hectáreas, la gran deforestación de la mencionada provincia es su mayor amenaza puesto que las precipitaciones y su clima han cambiado de radical manera. En esmeraldas, precisamente en el cantón Quinindé, se encuentra un sin número de sembríos de palmas africanas, en sitios donde anteriormente eran bosques tropicales, por ello se deduce a que estas plantaciones están causando un impacto ambiental en Quinindé y el mundo.

La presente investigación tiene como objetivo determinar los impactos generados por el derrame de lodo hacía el río blanco y a la población. En la presente investigación fue relevante la utilización de medios bibliográficos para la obtención de información y poder llevar a cabo nuestra temática planteada, además de la elaboración de un levantamiento de encuesta realizada a moradores del sector La Independencia. A continuación se detallará los métodos que fueron utilizados para el presente trabajo.

Método Exploratorio. - En el presente trabajo se realiza una revisión de los fundamentos y principales procedimientos del análisis factorial exploratorio, un método esencial para la construcción, adaptación y validación de este artículo. El artículo se presenta considerando las principales decisiones estratégicas que debe tomar el investigador durante la implementación del método. Se discuten además las distintas opciones conceptuales y metodológicas que se plantean en cada fase de la aplicación del Método Exploratorio.

Método Descriptivo. - En este método se realiza una exposición narrativa, numérica y/o gráfica, bien detallada y exhaustiva de la realidad que se estudia. El método descriptivo busca un conocimiento inicial de la realidad que se produce de la observación directa del investigador y del conocimiento que se obtiene mediante la lectura o estudio de las informaciones aportadas por otros autores. Se refiere a un método cuyo objetivo es exponer con el mayor rigor metodológico, información significativa sobre la realidad en estudio con los criterios establecidos por la academia.

Método analítico.- Está basado en experimentación lógica, directa y empírica, se lo utiliza en ciencias sociales y naturales, además este método es de gran importancia puesto que se lo utiliza en estudios descriptivos y nos permite aprender de aciertos experimentales como de sus errores.

Método Cuantitativo.-Es aquella en la que se recogen y analizan datos cuantitativos sobre variables. La investigación cualitativa evita la cuantificación. Los investigadores cualitativos hacen registros narrativos de los fenómenos que son estudiados mediante técnicas como la observación participante y las entrevistas no estructuradas. La diferencia fundamental entre ambas metodologías es que la cuantitativa estudia la asociación o relación entre variables cuantificadas y la cualitativa lo hace en contextos estructurales y situacionales. La investigación 
cualitativa trata de identificar la naturaleza profunda de las realidades, su sistema de relaciones, su estructura dinámica. La investigación cuantitativa trata de determinar la fuerza de asociación o correlación entre variables, la generalización y objetivación de los resultados a través de una muestra para hacer inferencia a una población de la cual toda muestra procede. Tras el estudio de la asociación o correlación pretende, a su vez, hacer inferencia causal que explique por qué las cosas suceden o no de una forma determinada.

\section{DESARROLLO}

Quinindé es una entidad territorial subnacional perteneciente a la Provincia de Esmeraldas. Su economía se ha basado en la agricultura, ganadería, industria y comercio, La ganadería de doble propósito, es el complemento de la riqueza, con la producción de carne y leche. Su cabecera cantonal es la ciudad de Rosa Zárate, lugar donde se agrupa gran parte de su población total. De los 150.000 habitantes alrededor de 63.000 constituyen la población de votantes.

Para el año 1990 Quinindé contaba con una población de 62.680 habitantes, con el paso del tiempo su población fue creciendo y ya en la década del 2001 según el censo realizado en ese año la población aumentó a 88.337 habitantes, trasladándonos al año 2010 Quinindé ya contaba con una población de 122.570 (Hora s. f.).

Este cantón ha ido creciendo paulatinamente, en la actualidad cuenta con importantes industrias que procesan variedades de aceites, en especial el de la palma africana, y es así como Quinindé mantiene su economía. El sitio donde ocurrió el derrame de lodo activado tiene como nombre La Independencia, el lugar es una jurisdicción territorial que en la actualidad cuenta con 7.000 habitantes perteneciente a la parroquia La Unión, del cantón Quinindé, que por su ubicación geográfica está ubicada en el límite de la provincia de Esmeraldas con Pichincha y Santo Domingo de los Tsáchilas y está conformado por 12 barrios: cuatro que pertenecen al centro poblado y los 8 restantes son considerados barrios urbanos, de la cabecera cantonal Rosa Zárate (Quiñonez, 2020).

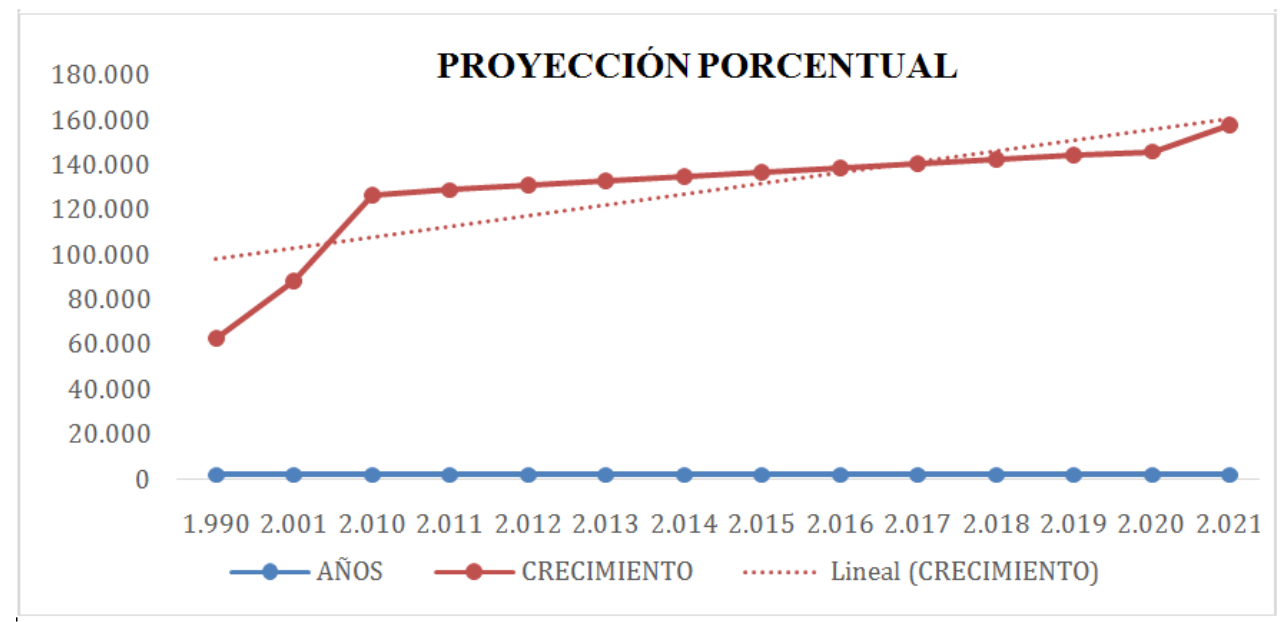

Figura 1: Proyección porcentual.

196 UNESUM-Ciencias. Publicación cuatrimestral. Vol. 4, No. 4 (Septiembre-Diciembre), Año 2020. 


\section{Generalidades de la palma africana}

La palma africana es una planta tropical, originaria de África, se la usa para obtener aceite, su propagación inició en el Siglo XV. En Ecuador la plantación de esta palma por lo general se la realiza en zonas boscosas húmedas tropicales con una temperatura que va desde los 24 a $28^{\circ} \mathrm{C}$. Este cultivo se adapta de mejor manera en la franja ecuatorial ya que aquí son estables las condiciones ambientales. El cultivo de palma africana ha generado una gran importancia económica a nivel mundial puesto a que los derivados que se logra obtener de esta se expenden por todo el planeta. Estas plantaciones han crecido considerablemente, esto se debe a la gran demanda que han tenido las palmas africanas de aceite en especial por los países del norte para producir cosméticos, lubricantes, entre otros. Es importante mencionar que Países de América Latina contienen grandes hectáreas sembradas, en el caso de Ecuador cuenta con 280 mil.J. (2019, 24 julio).

\section{Afectaciones al medio ambiente}

La ruptura del GeoTubo contenedor de lodos sedimentados en el recinto La Independencia, de la parroquia La Unión, cantón Quinindé, por parte de la extractora SOPALIN S.A originó una prominente contaminación del río blanco causando que este se vea perjudicado por el derrame, afectando a las especies de este ecosistema. Cabe mencionar que este lodo contenía restos de aceites en especial el de la palma africana, la cual también es un indicador que altera el medio ambiente y acuático (Anón s. f.).

\section{Daños al medio ambiente, acuático y terrestre}

La manera excedente en que se ha estado cultivando la palma africana ha generado la destrucción de bosques tropicales, emisiones como el co2 se han incrementado y además ha deteriorado zonas de cultivo. Un estudio realizado por ICTA-UAB en Guatemala arrojó como resultado que las plantaciones de palmas africanas han estado sustituyendo en un $40 \%$ bosques tropicales. Investigadores se han pronunciado y han llegado a la conclusión de que los suelos que son participes para producción de palma africana podrían durar al menos veinticinco años en ser fértiles nuevamente.

Como se dijo anteriormente una de las consecuencias ambientales que produce este monocultivo de palma africana es la infertilidad del suelo, ya que absorbe nutrientes en abundancia, a esto se añade que el suelo queda demasiado débil, y aunque se le añada abono, estos componentes desaparecen dando como resultado un suelo no fértil, otro problema a generarse por esta plantación es que elimina la capa orgánica del suelo (Mingorria Sara).

Otra de las consecuencias que se dan por estas plantaciones de palma africana es el daño que provoca a los ríos, por lo general suelen plantarse cerca de zonas donde exista gran cantidad de agua, como en el caso del río blanco, en Quinindé; alrededor del afluente están ubicadas las palmas africanas y del mismo modo la extractora causante del derrame de lodo activado hacía este ecosistema, como es el río blanco. De acuerdo a datos de naciones unidas la palma africana posee una alta huella hídrica de $5000 \mathrm{~m} 3$ por tonelada. Las plantaciones de la palma aceitera requieren del uso de agroquímicos, como es el caso del malatión, el cual es un plaguicida, y se lo usa para la eliminación de insectos como lo son las moscas que posan en la fruta de la palma. Por 
William J. Paredes Álava, Lisseth S.Vásquez Alarcón, Genny D. Cortez Quiñonez, Luis F. Lucio Villacreses

lo tanto al utilizar constantemente estos tipos de agroquímicos lo que harán estos es contaminar fuentes de agua, causándole no solo daño al río sino a las especies que habitan en dicho medio.

De acuerdo a los análisis comparativos de suelos realizados por la investigadora Sandra Mingorria se señala que la vida útil de la palma africana es de veinticinco años, después de este período se debe sustituir la plantación eliminándola y fertilizando el suelo para luego volver a plantar, esto requiere de un precio alto, y además de no ser rentable, el suelo apenas empieza a recuperarse. Por estas acciones es que las grandes industrias deciden emprender una búsqueda en sitios diferentes, ya sea en terrenos baldíos o bosques y así generar plantaciones nuevas de esta palma de aceite africana. Si bien es cierto que estas acciones, como lo es la plantación de palma africana, pueden plantarse de manera sostenible, dando lugar a bacantes de trabajo. Sin embargo se debe tomar en cuenta que estas plantaciones pueden llegar a generar un gran desastre ambiental.

\section{Principales impactos al medio ambiente}

Uno de los daños que ocasionan las plantaciones de palma africana es la deforestación. El programa de naciones unidas para el medio ambiente (PNUMA) deduce que se ha creado de manera ilegal plantaciones de palma africana en parques nacionales que resultan pertenecer a áreas protegidas.

La Universidad de Princeton y el Instituto Federal Suizo de Tecnología, calculan que en los años 1990 a 2005 hubo del 55\% a 60\% de expansión de este cultivo en Malasia e Indonesia. Otro dato importante señalado por el PNUMA fue que para el 2007 la palma africana fue la causante principal de deforestación en los países antes mencionados (Consumers.f).

\section{Pérdida de biodiversidad}

La palma africana se siembra en sitios tropicales, donde exista gran húmedas, y traen consigo un impacto ambiental puesto a que ellos provocan que las especies habitantes de bosques se vean en la necesidad de desplazarse, además de causar daño a puntos calientes de biodiversidad.

\section{Contaminación del aire y el aumento del cambio climático}

La quema de bosques para el sembrío de plantaciones de palma africana produce que se libere Co2 a la atmósfera, provocando que esta se vea polucionada por los gases de efecto invernadero que se emiten en el humo producto de la quema, generando que contribuyan al calentamiento global. La investigación abarca los objetivos de desarrollo sostenible. Es relevante mencionar que son 21 los ODS, pero el presente trabajo hace enfoque a los siguientes.

\section{Relación de la temática investigada con los objetivos de desarrollo sostenible}

Objetivo 8: Trabajo decente y crecimiento económico.

Con este objetivo lo que se busca es promover el crecimiento económico sostenible, empleos dignos para toda persona.

Meta 8.4: Mejorar progresivamente, de aquí a 2030, la producción y el consumo eficientes de los recursos mundiales y procurar desvincular el crecimiento económico de la degradación del medio ambiente, conforme al Marco Decenal de Programas sobre Modalidades de Consumo y Producción Sostenibles, empezando por los países desarrollados. 
Objetivo 11: Ciudades y comunidades sostenibles.

El presente objetivo propone en lograr que las personas vivan en un entorno o ambiente seguro, sostenible, sustentable y resiliente.

$>$ Meta 11 b: De aquí a 2020, aumentar considerablemente el número de ciudades y asentamientos humanos que adoptan e implementan políticas y planes integrados para promover la inclusión, el uso eficiente de los recursos, la mitigación del cambio climático y la adaptación a él y la resiliencia ante los desastres, y desarrollar y poner en práctica, en consonancia con el Marco de Sendai para la Reducción del Riesgo de Desastres 2015-2030, la gestión integral de los riesgos de desastre a todos los niveles.

Objetivo15: Vida de ecosistemas terrestres.

Las actividades humanas han generados daños al medio ambiente por lo que el presente objetivo busca promover la desertificación y detener la pérdida de diversidad biológica.

> Meta 15.1: De aquí a 2020, asegurar la conservación, el restablecimiento y el uso sostenible de los ecosistemas terrestres y los ecosistemas interiores de agua dulce y sus servicios, en particular los bosques, los humedales, las montañas y las zonas áridas, en consonancia con las obligaciones contraídas en virtud de acuerdos internacionales

\section{Datos Generales de la extractora SOPALIN S.A}

Tabla 1. Datos Generales de la extractora SOPALIN S.A

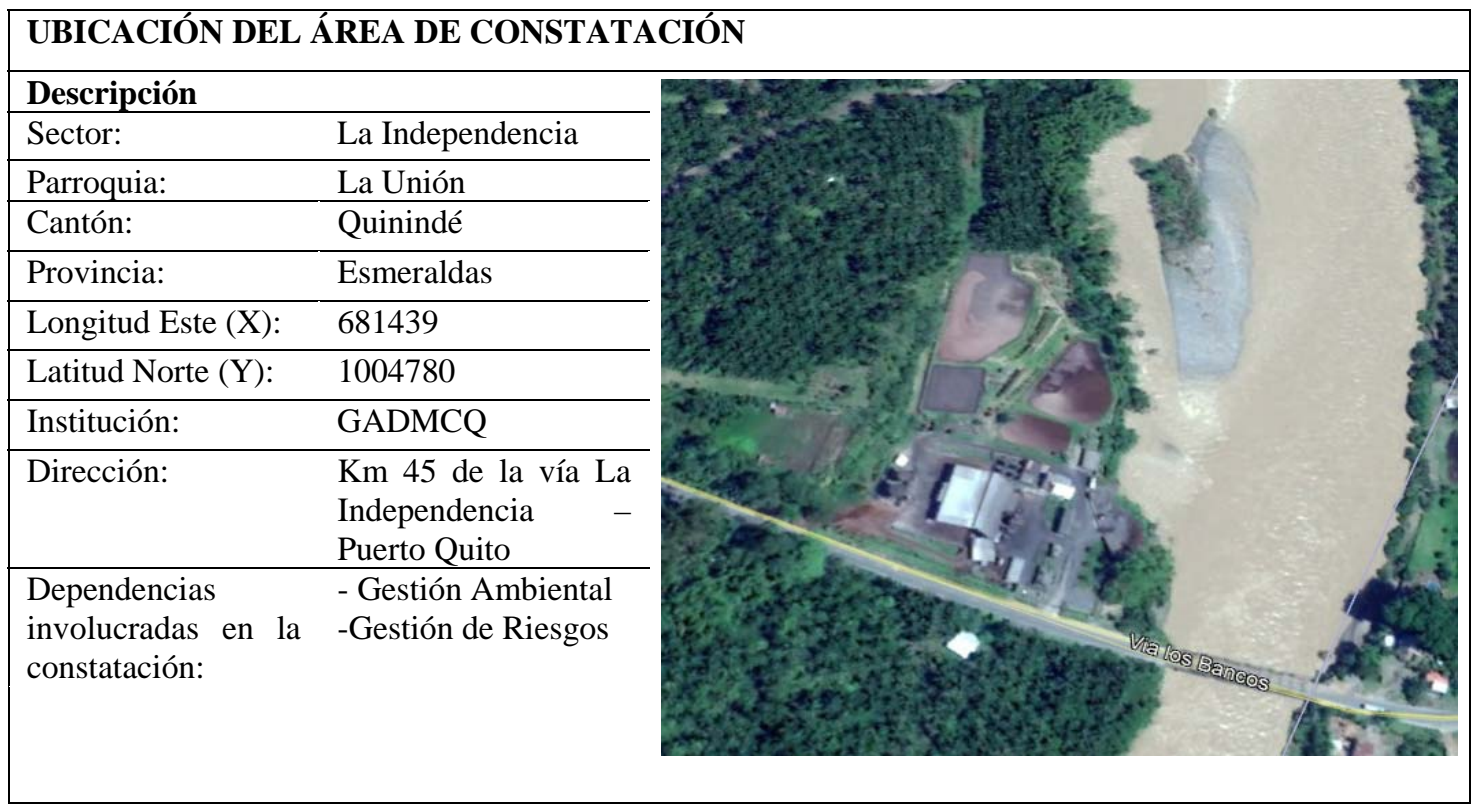


En el año 1988, cuando Industrias Ales, con don Francisco Uribe en la Presidencia, Wolfang Klein en la Gerencia y José Enríquez en la Dirección Financiera, deciden con la autorización de Don Patricio Álvarez Drouet, en ese entonces Embajador del Ecuador en los Estados Unidos, organizó la operación Santo Domingo, para solucionar el problema de comprar materia prima y ampliar su operación a nivel nacional. El reconocimiento del campo indica que para copar la zona era necesaria la presencia de la empresa tanto en la vía Quinindé como Quevedo, es así como se define por SOPALIN y Olepsa de prioridad en la adquisición.

La Sociedad de Palmicultores de la Independencia, SOPALIN S.A., había sido fundada por un grupo de profesionales agricultores el 12 de Julio de 1985, siendo su principal accionista Mariano Cedeño localizada a orillas del Rio Blanco, tenía una posición estratégica privilegiada para captar la mayoría de fruta de las plantaciones que se habían ubicado en la zona de la Concordia, la independencia, La Unión y la vía Puerto Quito (Esteves, 2020).

SOPALIN S.A se encuentra ubicada en el Km 45 de la vía La Independencia - Puerto Quito. Compra el $100 \%$ de la fruta que procesa (aceite rojo). Su capacidad instalada es de 14 ton/hora y efectivas en proceso 12 ton/hora. Su único cliente es Industrias Ales.

\section{RESULTADOS}

Los resultados que se presentan a continuación toman información del departamento de medio ambiente del municipio de Quinindé, GAD Municipal de Quinindé y de la encuesta realizada por el equipo de investigación a 21moradores del recinto La independencia de la parroquia La Unión del Cantón Quinindé, sitio afectado debido a la ruptura del geo tubo contenedor de lodos sedimentados, por parte de la extractora SOPALIN S.A.

La comunidad en un 90,5\% dio a conocer que el derrame producido por la extractora SOPALIN S.A originó una contaminación media alta. Cuya afectación fue visible en el tema paisajístico, con una cantidad de 30000 yd $^{3}$, según el (GAD) de Quinindé.

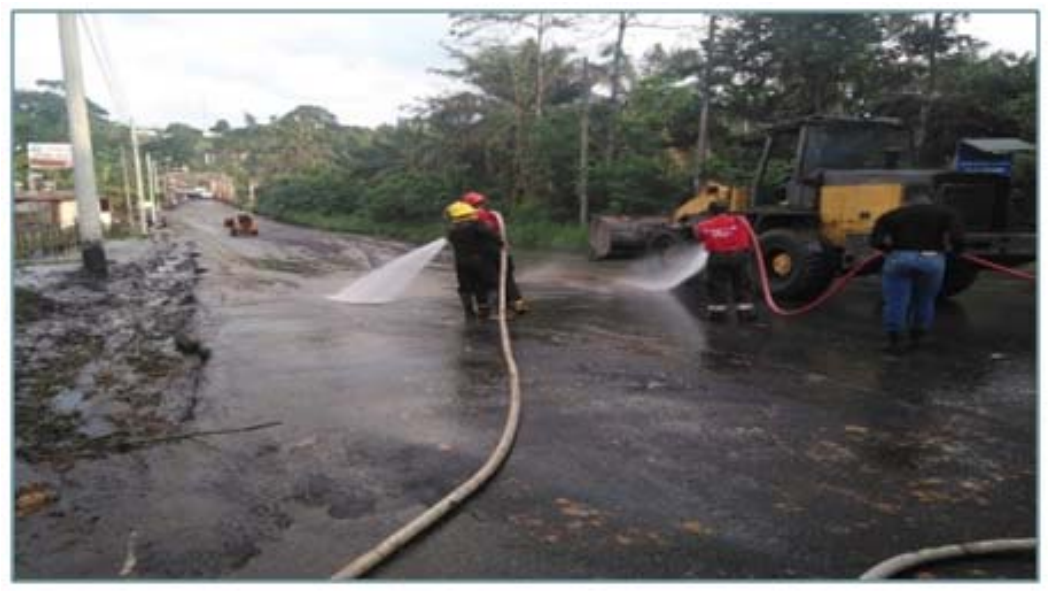

Figura 2. Lavado de vías afectadas con ayuda del cuerpo de bomberos del cantón Imagen Tomada: por el departamento de ambiente del GAD de Quinindé 
Mediante este gráfico realizado por el equipo de investigación se da a conocer que un 42,9\% de la comunidad desconoce las medidas para evitarla contaminación del agua. Y por lo tanto no miden el daño que podrían ocasionarle al afluente ya sea por alguna otra fuente de contaminación.

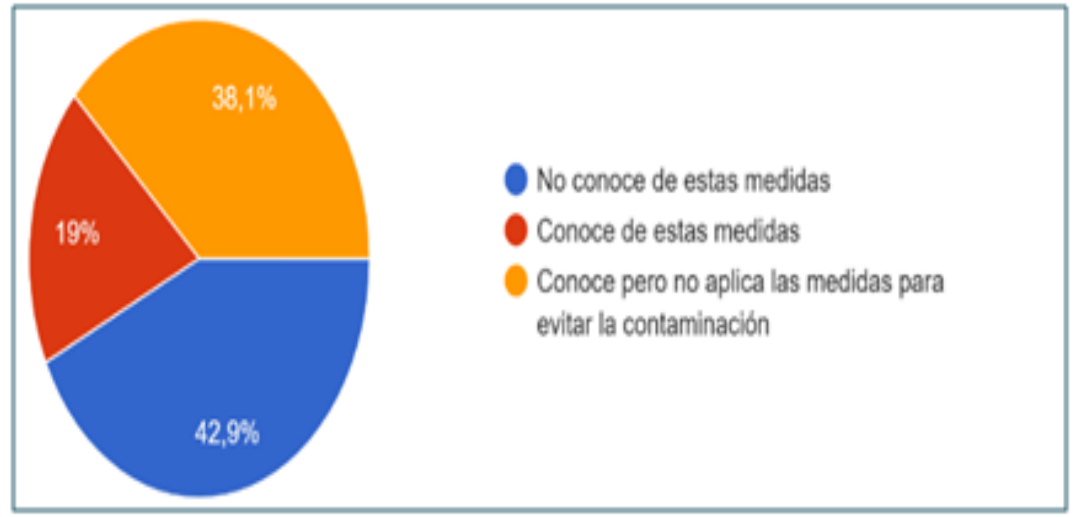

Figura 3. Gráfico sobre la contaminación del agua Elaborado por Equipo de investigación

La población evidenció un 71,4\% de afectación en la flora y fauna, generándose una extensa cantidad contaminada de la zona.

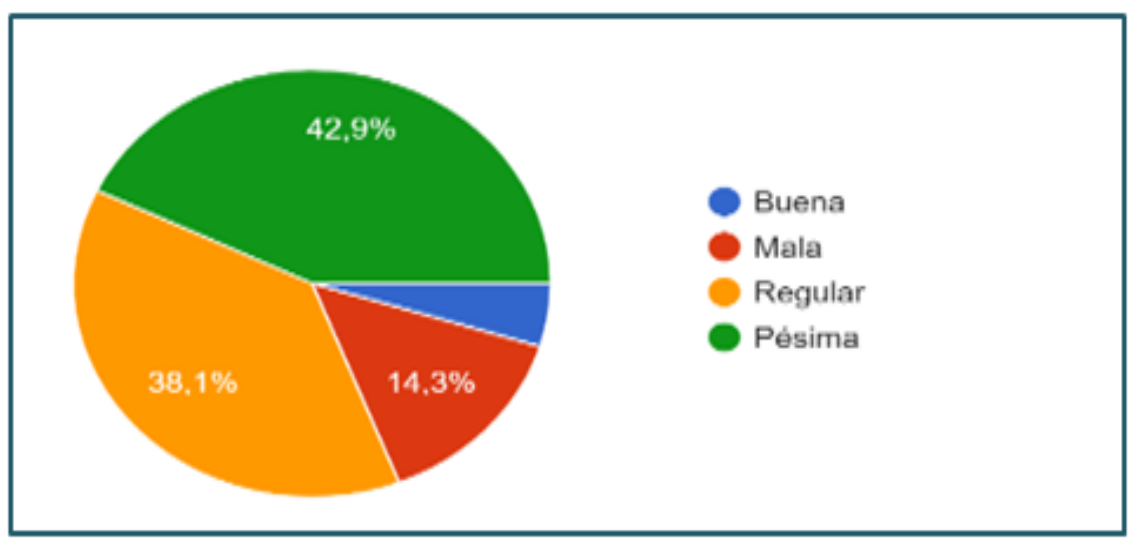

Figura 4. Gráfico 3 sobre los daños a la flora y fauna Elaborado por Equipo de investigación

Mediante el estudio realizado por el equipo de investigación se dio a conocer que el 71,4\% del agua del río blanco aún contiene sustancias de lodo activado las cuales fueron disueltas por parte del cuerpo de bomberos del cantón Quinindé y la Concordia, quienes utilizaron la cantidad de dos tanqueros de agua para remover el lodo derramado enviándolo directamente al río. 


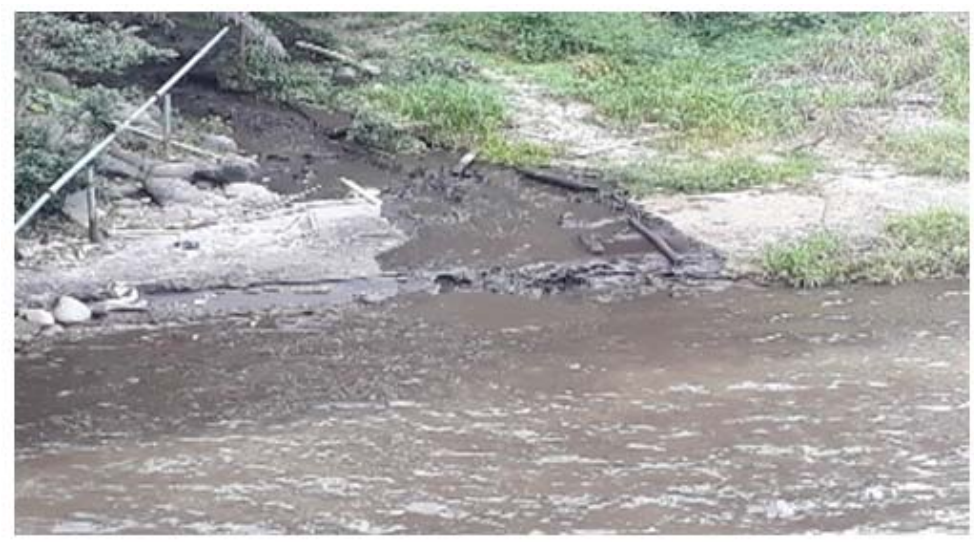

Figura 5. Sedimentos arrojados al río

Imagen Tomada: por el departamento de ambiente del GAD de Quinindé

Según el equipo de investigación se aplicó parcialmente la legislación ambiental, sin embargo la empresa SOPALIN S. A no brindó mantenimiento adecuado al geo tubo lo que provocó un colapso del mismo , generando derrame de lodo activado, viéndose afectado el ecosistema terrestre, acuático y la población.

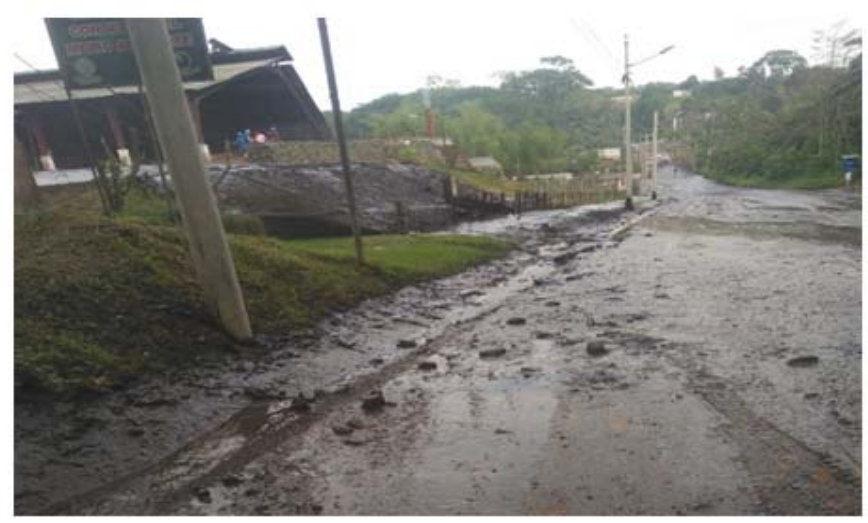

Figura 6. Lodo derramado en la vía Imagen Tomada: por el departamento de ambiente del GAD de Quinindé

El equipo de investigación determinó que el 85,7\% de cultivo de palma africana ha generado un desplazamiento de flora, fauna y un impacto ambiental de la zona. Este monocultivo provoca infertilidad en el suelo ya que succiona nutrientes en grandes cantidades, además de contaminar el agua, deteriora el horizonte del suelo. 


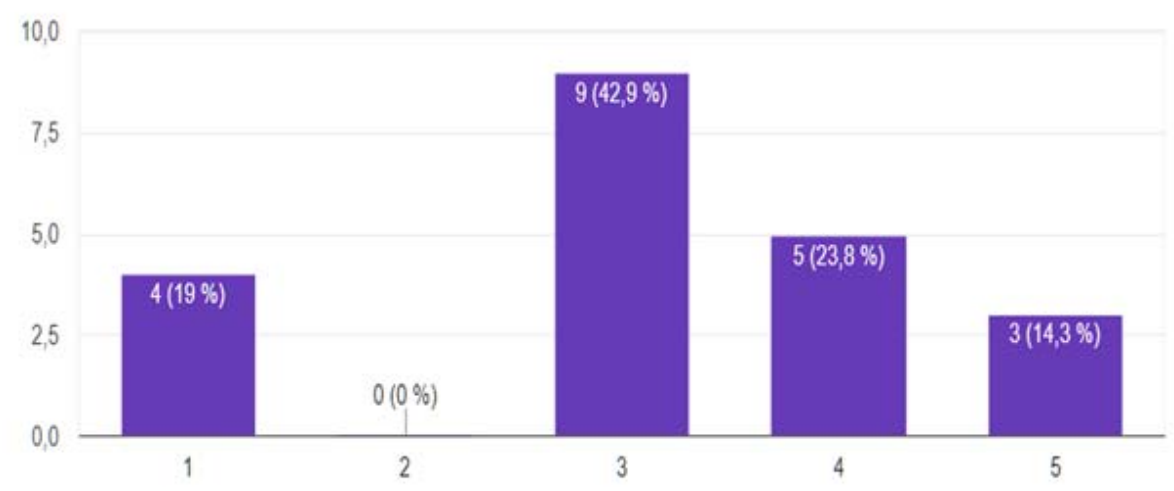

Figura 7. Gráfico sobre la Palma africana y su impacto ambiental

La comunidad, en un 57,1\%, dio a conocer que está al tanto sobre la responsabilidad que tiene la empresa con sus empleados y habitantes, en caso de presentarse un incidente donde se vea afectada una comunidad y el medio ambiente. El derrame de lodo activado, cumpliendo de tal manera con el Art. 14 de la Constitución de la República donde se especifica el derecho de la población a vivir en un ambiente sano y ecológicamente equilibrado, que garantice la sostenibilidad y el buen vivir causado por la extractora de aceite SOPALIN S.A, perjudicó a moradores, flora y fauna; trayendo consigo un problema económico y social, pese a esto la empresa tuvo la obligación de responder por lo acontecido, con el fin de eliminar la contaminación generada

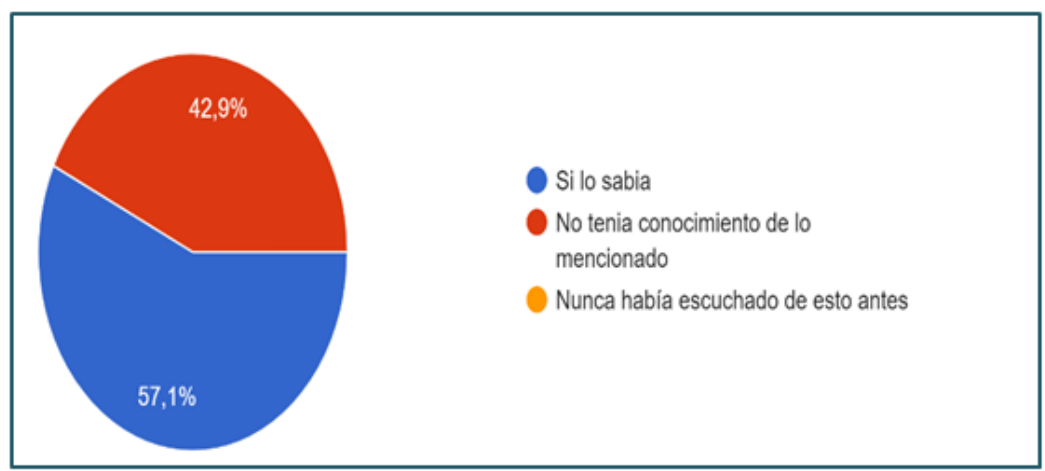

Figura 8. Gráfico sobre el compromiso de sostenibilidad y buen vivir de empresa hacia comunidad

\section{CONCLUSIONES}

La rotura del geo tubo de la extractora SOPALIN S.A., causó impacto paisajístico ya que hubo una gran cantidad de lodo sedimentado en la vía y el mismo desembocó al río Blanco causándole una contaminación media alta de $30000 \mathrm{yd}^{3}$, según el informe N.- 027-2019-JGA-GADMCQ: constatación del derrame en la extractora SOPALIN S.A. 
William J. Paredes Álava, Lisseth S.Vásquez Alarcón, Genny D. Cortez Quiñonez, Luis F. Lucio Villacreses

Si la empresa cumple con los objetivos de desarrollo sostenible planteados en la investigación, pese haberse suscitado el incidente, puede proporcionar trabajo para la comunidad y cumple con las normativas ambientales. La investigación permitió conocer que SOPALIN S.A con la responsabilidad social corporativa, ya que cuenta con los permisos requeridos y normas ambientales para llevar a cabo sus actividades laborales.

\section{REFERENCIAS BIBLIOGRÁFICAS}

A. (2019a, julio 20). Aceite de palma: así daña al medio ambiente. Consumer. Obtenido de https://www.consumer.es/medio-ambiente/aceite-de-palma-asi-dana-al-medio-ambiente.html

Anón. s.f. «Aceite de palma | OCU». www.ocu.org. Recuperado 14 de agosto de 2020 (https://www.ocu.org/alimentacion/alimentos/informe/aceite-de-palma).

Anón. s. f. «Por derrame de aceite estuvo cerrada por varias horas la vía La Independencia - Puerto Quito | Ecuador | Noticias | El Universo». Recuperado 12 de agosto de 2020. Obtenido de

(https://www.eluniverso.com/noticias/2019/11/14/nota/7604540/derrame-aceite-estuvo-cerrada-varias-horasindependencia-puerto).

Barcelona, M. A. (2017, 6 abril). El aceite de palma es una de las principales causas de deforestación del planeta. elperiodico. Obtenido de

https://www.elperiodico.com/es/medio-ambiente/20170405/aceite-de-palma-principales-causas-deforestacionplaneta-5953684

Barcelona, U.-U. A. (2017). Las plantaciones de palma aceitera provocan la infertilidad de los suelos tropicales. UAB Barcelona. Obtenido de

https://www.uab.cat/web/sala-de-prensa/detalle-noticia/las-plantaciones-de-palma-aceitera-provocan-la-infertilidadde-los-suelos-tropicales-1345667994339.html?noticiaid=1345727879056

CONSUMER (https://www.consumer.es/), EROSKI. s.f. «Aceite de palma: así daña al medio ambiente». Recuperado 12 de agosto de 2020 (https://www.consumer.es/medio-ambiente/aceite-de-palma-asi-dana-almedio-ambiente.html). Obtenido de https://www.consumer.es/medio-ambiente/aceite-de-palma-asi-dana-almedio-ambiente.html

Hora, Diario La. s. f. «Quinindé crece en población y desarrollo - La Hora». La Hora Noticias de Ecuador, sus provincias y el mundo. Recuperado 12 de agosto de 2020. Obtenido de https://lahora.com.ec/noticia/1000104481/quinind-crece-en-poblacin-y-desarrollo.

J. (2019, 24 julio). La palma africana se apodera silenciosamente de la Amazonía de Ecuador. Noticias ambientales. Obtenido de https://es.mongabay.com/2017/10/ecuador-palma-africana-en-la-amazonia-

lalineadefuego.info. (2018, 11 julio). LA PALMA ACEITERA EN EL ECUADOR: ¿UN CULTIVO SOCIAL Y SUSTENTABLE? Por Geovanna Lasso*. WordPress.com. Obtenido de.

https://lalineadefuego.info/2018/07/10/la-palma-aceitera-en-el-ecuador-un-cultivo-social-y-sustentable-por-

geovanna-lasso/Municipalidad de Quinindé. (2019). INFORME N.- 027-2019-JGA-GADMCQ: Constatación del derrame en la extractora sopalin S.A

Quiñonez (comunicación personal 14 de Agosto, 2020)

204 UNESUM-Ciencias. Publicación cuatrimestral. Vol. 4, No. 4 (Septiembre-Diciembre), Año 2020. 